\title{
EchoGéo
}

$7 \mid 2008$

Les nouveaux enjeux régionaux dans l'océan Indien occidental

\section{Madagascar au XXIe siècle : la politique de sa géographie}

Hervé Rakoto Ramiarantsoa

\section{OpenEdition}

Journals

Édition électronique

URL : https://journals.openedition.org/echogeo/8753

DOI : 10.4000/echogeo.8753

ISSN : 1963-1197

Éditeur

Pôle de recherche pour l'organisation et la diffusion de l'information géographique (CNRS UMR 8586)

Référence électronique

Hervé Rakoto Ramiarantsoa, « Madagascar au XXle siècle : la politique de sa géographie », EchoGéo

[En ligne], 7 | 2008, mis en ligne le 27 octobre 2008, consulté le 31 juillet 2021. URL : http://

journals.openedition.org/echogeo/8753; DOI : https://doi.org/10.4000/echogeo.8753

Ce document a été généré automatiquement le 31 juillet 2021.

EchoGéo est mis à disposition selon les termes de la licence Creative Commons Attribution - Pas

d'Utilisation Commerciale - Pas de Modification 4.0 International (CC BY-NC-ND) 


\title{
Madagascar au XXIe siècle : la politique de sa géographie
}

\author{
Hervé Rakoto Ramiarantsoa
}

\section{Introduction}

1 Dans quelle mesure la "politique de sa géographie ", formule utilisée par le président Ratsiraka lors de son mandat de 1975 à 1992, change-t-elle la donne de la question du développement, toujours au coeur des préoccupations d'une nation classée dans le groupe des Pays Pauvres et Très Endettés?

2 L'expression "politique de sa géographie » présente deux significations La première s'entend dans un sens géopolitique. C'est le cas à la fin des années 1980 lorsque le président Ratsiraka annonce un changement de cap de son gouvernement, esquissant un rapprochement avec l'Afrique du Sud ; cette dernière était pourtant sous embargo de l'Organisation de l'Unité Africaine à cause de sa politique d'apartheid. L'initiative n'est pas nouvelle, elle continue des contacts discrets, menés sous la Première République (1960-1972). Raison (1995) montre comment Madagascar a toujours valorisé sa position géographique en tirant parti des tensions internationales : le Sud Ouest de l'océan Indien correspond en effet plus à un interface entre différents systèmes, qu'il ne forme un véritable système régional à personnalité propre. L'affirmation de l'Afrique du Sud comme puissance régionale depuis la fin des années 1990 modifie-telle la donne? L'adhésion de Madagascar à la Communauté de développement de l'Afrique australe (Sadc, Southern Africa Development Community) en 2005 confirme en tout cas l'intérêt que porte le pays aux bénéfices engendrés par sa situation géographique. "Politique de sa géographie » se décline aussi suivant une seconde acception. Il s'agit de l'exploitation des ressources dont le pays dispose, étant entendu qu'une ressource n'existe pas dans l'absolu: elle est définie en tant que telle en fonction de l'usage auquel elle est destinée. Un élément ne devient ressource que par ce que les hommes en font (Gourou, 1982; Pélissier, 1995 ; Dupré, 1996). Ainsi, dans le contexte mondial actuel des gisements miniers, les chantiers d'Ambatovy (nickel 
cobalt) et de Tolagnaro (ilménite) s'avèrent intéressants pour devenir ressources en vue du développement.

3 La réflexion que je propose mobilise le thème des relations entre développement et environnement, à travers deux types de ressources dont la richesse distingue la grande île, les ressources agricoles et celles de la biodiversité. Si l'agriculture marque ce Pays Très Rural (Ptr : plus de $75 \%$ de la population se définit comme paysanne et vit dans les campagnes) avec une très large palette de cultures équatoriales, tropicales et tempérées. Une "mégabiodiversité » avec un taux d'endémicité élevé distingue Madagascar (Goodman et Benstead, 2004). L'utilisation de ces deux types de ressources intègre des considérations nationales, car il s'agit de réalités importantes pour des populations ou pour des filières intéressant différents niveaux d'acteurs, et supranationales, dans la mesure où dynamiques locales et mécanismes englobant interfèrent en permanence.

4 La réflexion souligne les difficultés de mener une politique de sa géographie pour un pays qui voit les exigences de la mondialisation parfois s'accorder avec, parfois contredire, les objectifs de développement durable des sociétés et de leurs territoires. Elle évoque dans un premier temps le contexte de modernité dans lequel Madagascar conçoit son développement. Elle se penche dans une deuxième partie sur les questions que posent les interférences des préoccupations environnementales avec les enjeux socio-économiques, et interroge dans un dernier temps la pertinence du modèle de développement agricole choisi.

\section{Une modernité mise au service du développement}

5 Telle qu'elle est apportée par la globalisation et son système de mise en relation des territoires et des hommes, la modernité du XXI ${ }^{e}$ siècle touche Madagascar. Elle se traduit dans des domaines très variés qui concernent les réalités quotidiennes, les paysages urbains et ruraux, les modes d'administration du pays...

6 La capitale Antananarivo en reflète plusieurs aspects. On peut noter les grands axes routiers récents, aux noms évocateurs de ceux qui en ont assuré le financement ${ }^{1}$ (avenue de l'Europe, boulevard de Tokyo...). Ces larges voies urbaines traversent, desservent ou contournent la cité ; elles améliorent l'accès à l'agglomération qui a très largement dépassé le million d'habitants. Il faut aussi relever une politique urbaine inscrite dans le courant de l'écologisation des villes à la fin des années 1990. Une telle disposition a abouti à la disparition du grand marché central du zoma, faisant d'Antananarivo une ville "sans âme", selon l'expression du très populaire chanteur Eric Manana. Cependant, elle met en valeur un des charmes de l'agglomération, son agriculture intra et périurbaine (Université d'Antananarivo, 2007) qui participe de la nature « verte» de la ville.

7 La valorisation des ressources du sous-sol représente une autre forme de modernité. Les deux gros projets d'exploitation minière d'Ambatovy, dans la partie centre orientale de l'île, et de Tolagnaro dans le sud-est, apportent des infrastructures et des aménagements qui intègrent dans l'économie nationale, des secteurs autrefois marginaux. Il en est de même pour la présence d'hydrocarbures, que l'évolution des prix à l'échelle mondiale met au premier plan; la production sur le site on shore de Tsimiroro (versant centre ouest de l'île) doit ainsi commencer en 2010. De telles exploitations s'avèrent par ailleurs être un moyen efficace pour attirer les 
Investissements Directs à l'Etranger (IDE) et tirer parti des apports technologiques, financiers, sociaux de ces derniers.

Enfin, l'engagement des autorités malgaches dans les processus de transfert de gestion des ressources naturelles auprès de communautés qui en sont riveraines, est une autre disposition très moderne. Il répond, certes, à la recherche d'une " amélioration des modes de gestion" (termes du document d'orientation de la politique nationale forestière, votée en 1997) en responsabilisant les acteurs locaux. La loi de 1996 sur la participation des associations paysannes à l'administration de telles ressources va dans ce sens ; Kull (1996) en note un objectif, "to end the spiral of degradation by reconciling the population with the environment" Cet engagement n'en met pas moins au premier plan des principes chers à la bonne gouvernance, que Michon (2002) évoque ainsi : « participation, gouvernance locale... deviennent les maîtres mots du nouveau discours idéologique... ».

9 Cette modernité est mise au service du développement d'une nation qui combine au paradigme prégnant du développement durable, la nécessité d'un développement rapide (Plan d'action Madagascar, 2006). Quelle place la lutte contre la pauvreté accorde-t-elle alors à l'environnement, élément désormais angulaire des politiques publiques?

\section{L'environnement, une ressource pour le développement?}

10 La mégabiodiversité de Madagascar place la question environnementale au centre de différents enjeux. Enjeu scientifique, car l'île présente tous les types de flores présents ailleurs sous les tropiques (Guillaumet, 1984) et sa richesse faunistique comporte des espèces considérées comme des « chaînon manquants "-c'est le cas avec les primates-, précieux pour établir les relations entre les groupes d'espèces paléotropicales (Goodman et Benstead, op. cit.). La diversité représente aussi un enjeu économique. Elle fonde un intérêt pour la bioprospection en même temps que ses éléments sont une ressource utilisée dans le système économique et territorial des populations locales. Il en résulte des enjeux de pouvoirs entre les différentes parties prenantes dès lors qu'il s'agit de parler des relations entre environnement et développement : représentants et services techniques de l'Etat, bailleurs de fonds, organisations non gouvernementales, universités (Missouri Botanical Garden...), mais aussi représentants des populations riveraines des ressources de la biodiversité, ou encore l'UNESCO.

11 Le Programme National d'Action Environnemental (Pnae), mis en place en 1990, met en présence ces divers niveaux et catégories d'acteurs. Il faut cependant noter deux points. Le premier se rapporte au rôle désormais marginal accordé aux services publics officiels (Ramamonjisoa, 2004). Le second point concerne l'approche participative, incontournable depuis le sommet de Rio et sa Convention sur la Diversité Biologique. Cette approche apparaît d'autant plus problématique -Blanc Pamard et Boutrais (2002) parlent $\mathrm{d}^{\prime}$ " illusion localiste »- que les modèles biologiques de conservation de la «vision Durban» (2003) demandent de grands espaces², ce qui nécessite une faible présence (donc une faible participation ?) humaine.

12 S'engager dans un Plan National d'Action Environnemental exprime une volonté de mise en ordre de la «nature», ce qui fondamentalement est un projet politique 
concernant l'accès des sociétés aux ressources de leurs territoires. Je présenterai dans un premier temps cette dimension du PNAE pour en souligner la philosophie, à travers le fonctionnement des institutions qu'il met en place. J'analyserai ensuite, à partir d'exemples tirés de quelques études de cas, quels types de rapports un tel projet établit entre les différents niveaux d'acteurs impliqués. En effet, selon leur nature (indifférence, alliance, opposition...), ces relations se trouvent au cœur de l'échec ou de la réussite de l'action. Elles posent la question de savoir dans quelle mesure l'environnement peut être une ressource pour le développement.

\section{Le Pnae, un projet politique}

13 Le constat de départ est celui d'une forte menace: Sahler (2005) note que, pour la Banque Mondiale, si le taux de déforestation de $1 \%$ par an ne change pas, les seules forêts restantes dans les cent prochaines années seront celles des réserves. La formule «Ile verte, île rouge » (Blanc-Pamard et Rakoto Ramiarantsoa, 2003) donne une image frappante de cette évolution et des effets conséquents de l'érosion.

L'Etat malgache a adopté en 1984 une "Stratégie nationale de conservation pour le développement », puis organisé l'année suivante une grande conférence internationale sur les problèmes environnementaux du pays, avec la participation du prince Philip, alors président du WWF. Ces dispositions, pionnières à l'échelle du continent africain, se situent dans un contexte mondial où la conservation des ressources naturelles devient un élément-clé de la gestion des territoires, inspirée par les Etats-Unis (Hufty, 2001). Elles s'appuient sur les grandes organisations du secteur mondial de la conservation (UICN, WWF) dont une des principales tâches est de conseiller les Etats, en « intervenant en amont des politiques publiques, en particulier en rédigeant les stratégies nationales de conservation » (Dumoulin et Rodary, 2005). Les mêmes auteurs font remarquer un « verdissement » de la coopération internationale à la fin des années 1980, avec la réorientation de la Banque Mondiale vers les problèmes de protection de l'environnement : les projetsdette-nature proposés aux États par le biais de WWF entrent dans ce cadre à Madagascar. Par ailleurs, Kull (1996) relève une nette croissance des investissements nord-américains pour les programmes de conservation de la biodiversité.

Il faut noter la place particulière des crédits américains dans le financement du PNAE 3 . L'USAID, agence bilatérale américaine d'aide au développement, assure $96 \%$ des besoins de la composante biodiversité entre 1991 et 1995 (Andriamahefazafy et Méral, 2003). Elle participe à hauteur de $50 \%$ au financement de l'ANGAP, principale organisation de gestion des Aires Protégées. Tout au long des trois phases du programme, l'appui financier des Etats-Unis reste prépondérant au sein d'un groupe de bailleurs (qui comprend l'Union Européenne, la Suisse, le Japon) dont la Banque Mondiale reste le principal coordinateur. On ne peut ne pas voir dans ces aspects budgétaires une prééminence des financeurs dans le contrôle des ressources de la biodiversité malgache.

Programme à sensibilité écologique, le PNAE traite le thème de la gestion de la nature pour en réglementer l'accès et l'usage des ressources: il s'agit bien d'un projet d'essence politique. Le choix de l'Etat malgache s'appuie sur des financements dont les conditionnalités peuvent apparaître comme une «traduction de normes internationales en applications nationales » (Aubertin, 2005). 


\section{Le PNAE à l'œuvre : des acteurs aux postures divergentes} différemment mises en œuvre selon les acteurs, ce qui traduit des problèmes d'articulation entre les intervenants. Ramamonjisoa (2004) observe que le WWF trouve plus urgent de préserver une "partie du patrimoine international» en freinant l'érosion de la biodiversité, que de lancer un véritable programme de développement rural durable. A l'autre bout de la chaine, à l'échelle locale, il constate le poids accordé aux transferts de gestion des ressources naturelles aux communautés riveraines, sans que pour autant des études scientifiques (inventaire systématique, données dendrologiques) soient menées pour connaître la «durabilité» de l'usage de la ressource et démontrer l'efficacité de cette forme de gestion. Le l'approche intégrée du PNAE répond à des objectifs différents, selon les niveaux des acteurs, et se trouve accompagné de relations qui, parce que « la participation ne porte pas sur le choix des outils, ni sur les objectifs qu'ilsservent » (Karsenty, 1998), produisent des résultats qui combinent peu environnement et développement.

Trois études de cas éclairent différemment une telle réalité. La première analyse une intervention de la FAO (Rakoto Ramiarantsoa, 1997b), la deuxième reprend les principaux éléments d'un transfert de gestion de ressources forestières (Rakoto Ramiarantsoa, Samyn, 2004), le dernier exemple livre des réflexions sur la conservation $\mathrm{du}$ corridor forestier betsileo (Blanc-Pamard et al., 2005).

\section{Le déphasage entre la FAO et les organisations paysannes locales}

Il s'agit d'une action de la FAO entrant dans le cadre des programmes de restauration et d'aménagement de bassin-versants (celui du Mahakamo, sur 3000 ha, dans la région d'Anjozorobe au nord-est de l'Imerina). L'objectif est d'«assurer la protection des facteurs de production que sont le climat, le sol et l'eau ..., viser la satisfaction des besoins intérieurs en bois ... et disposer d'un surplus exportable » (Randrianasolo et al., 1997). L'intervention doit mobiliser une approche participative "durant toutes les phases de son intervention en vue d'assurer les meilleures conditions de succès... ».

L'action comprend un volet foresterie (techniques et dimension foncière de reboisements), un volet agricole (aménagement conservatoire des terrains cultivés), un volet sylvo-pastoral (techniques, fourrage), ainsi que des micro-réalisations (ponts, puits, pistes). Les agriculteurs n'ont pas été indifférents à l'apport du projet, et en reconnaissent des points positifs : formation organisationnelle (associations, comités), nouvelles espèces végétales adoptées. Mais la rationalité technique proposée n'est pas $\mathrm{du}$ tout en accord avec les pratiques locales, qui expriment une adaptation des systèmes de production aux réalités du milieu. Le paysage révèle l'impact très ponctuel des aménagements du projet. Les paysans continuent d'exploiter préférentiellement des parties en pente du versant, contrairement aux objectifs de l'action : manifestement, il demeure une contradiction entre le souci de conservation des sols du projet et la logique du système agraire local. L'approche participative est en cause, car il n'y a pas eu « discussion du schéma avec les populations jusqu'à la finalisation du plan d'aménagement». Elle aurait permis de comprendre que les priorités locales se rapportent à des thèmes autres que ceux proposés. Ce décalage explique les résultats mitigés du programme. 


\section{Transfert de gestion de ressources forestières et empowerment des acteurs principaux}

21 de la gestion d'une forêt sacrée, Antsapia, toujours fréquentée pour différents rites et marque identitaire de sa région, située en bordure centre est des hautes terres. La phase 2 du PNAE privilégie de tels transferts auprès de communautés locales riveraines de la ressource.

La coopération suisse a assuré un accompagnement très actif pour la constitution, le dépôt, le suivi du dossier de demande de transfert. L'association formelle constituée pour cette demande compte uniquement des membres d'un groupe nobiliaire, celui des Andrianefintany déjà légitimé par ses relations privilégiées avec la forêt d'Antsapia. Le groupe contrôlait l'accès aux ressources d'Antsapia, et le transfert lui permet d'asseoir encore plus son autorité. Il y a, avec cette expérience, un véritable empowerment, et on peut se demander s'il faut s'en réjouir, car l'action renforce le contrôle de cette ressource forestière, ou au contraire y voir source de problèmes, car c'est aussi marginaliser encore plus les autres habitants de la région qui ne sont pas Andrianefintany : toute marginalisation est porteuse de conflits. Une analyse récente (Rakoto Ramiarantsoa, 2008) révèle que l'initiative de transfert se trouve détournée de son objectif de conservation initial, car les sociétés locales attendent désormais de l'engagement contractuel des perspectives de nature économique; le souci environnemental seul n'assure pas le développement.

\section{Le corridor forestier betsileo, une richesse locale spoliée en devenant bien national}

Long de plus de $160 \mathrm{~km}$, le corridor forestier Ranomafana-Andringitra-Ivohibe (RAI) est prioritaire pour la préservation « d'un massif forestier riche en biodiversité et menacé par les pressions humaines » (Blanc-Pamard, Rakoto Ramiarantsoa, Andriantseheno, 2005). Il fait l'objet d'un enchâssement de territoires de conservation, soit aires protégées, soit espaces dont la gestion des ressources naturelles a été transférée à des associations paysannes locales. Dans ce dernier cas, les contrats de gestion organisent un zonage de différents secteurs : protection, production, droits d'usage. Ils génèrent des dysfonctionnements lorsqu'ils font interférer espaces de conservation et formes d'organisation sociale et territoriale. Les zones de protection sont définies par des critères biologiques relevant $\mathrm{du}$ savoir scientifique universel. Elles signifient interdiction d'accès pour des groupes sociaux, claniques ou lignagers, qui en étaient historiquement propriétaires. La ressource devient interdite: «la forêt qui était la richesse » des populations locales échappe à ces dernières en se trouvant intégrée dans un corridor au statut d'aire protégée, et dont la conservation relève désormais d'un enjeu national, voire international, donc de règles d'une autre nature. La dépossessiongénère des réactions locales pour transformer les dispositifs réglementaires en opportunité technique, économique ou foncière. Se développent ainsi des pratiques de contournement, d'évitement, parfois de transgression plutôt que de recompositions par un zonage territorial, tel que le propose le contrat. Tout en étant significative de la non-participation des acteurs locaux à la définition de ces règles, 
cette dynamique révèle un sentiment de populations qui se considèrent comme spoliées de leurs ressources habituelles.

Même si les trois exemples sont limités à des situations sur les hautes terres, et même s'ils méritent d'être relativisés par d'autres situations où de tels dysfonctionnements sont secondaires par rapport à l'amélioration de l'état des ressources naturelles et de la qualité de vie locale, ils n'en soulèvent pas moins de manière convergente le problème de la primauté du souci environnemental sur les actions de développement rural. Si le président de la République a solennellement déclaré «notre gouvernement fera de la protection de notre précieux environnement une des priorités de nos priorités » lors de la $57^{\mathrm{e}}$ session de l'Assemblée Générale des Nations Unies, il s'agit bien d'un environnement au service du développement, ce qui est pourtant rarement le cas.

\section{Pour quel développement agricole?}

Les paysages agricoles malgaches révèlent une activité différenciée. Ils alternent des espaces de grands aménagements (cultures industrielles et périmètres irrigués des deltas de l'Ouest), des unités très modernes (ferme Tiko d'Andranomanelatra sur les hautes terres) des secteurs marqués par les abattis-brûlis (tavy du versant oriental, hatsake du Sud-Ouest), ou encore, beaucoup plus disséminés dans l'île, des terroirs de polyculture combinant plantes vivrières et commerciales. Cependant, le constat qui prime à l'échelle nationale est celui d'une « agriculture traditionnelle, peu intensive, très peu mécanisée et utilisant peu d'intrants » (Bernard et al., 2007). Il en découle un choix politique de modernisation pour rendre cette agriculture compétitive sur les marchés internationaux. Un tel choix se révèle problématique à l'égard des intérêts d'une agriculture familiale prédominante sur le territoire national.

\section{Modernisation, professionnalisation, marché : le Madagascar Action Plan (MAP) et la révolution verte...}

Le MAP (Madagascar Action Plan, ou Plan d'action Madagascar), document de base de la politique publique pour la période 2007-2012, met en valeur le rôle stratégique du secteur agricole. A ce dernier sont assignés des objectifs de croissance de la production et de développement agro-industriel. Le programme s'appuie sur des structures qui, dans le cadre de la décentralisation à l'oeuvre dans le pays, doivent contribuer à la réalisation d'une révolution verte durable (cf encadré 1 ). 


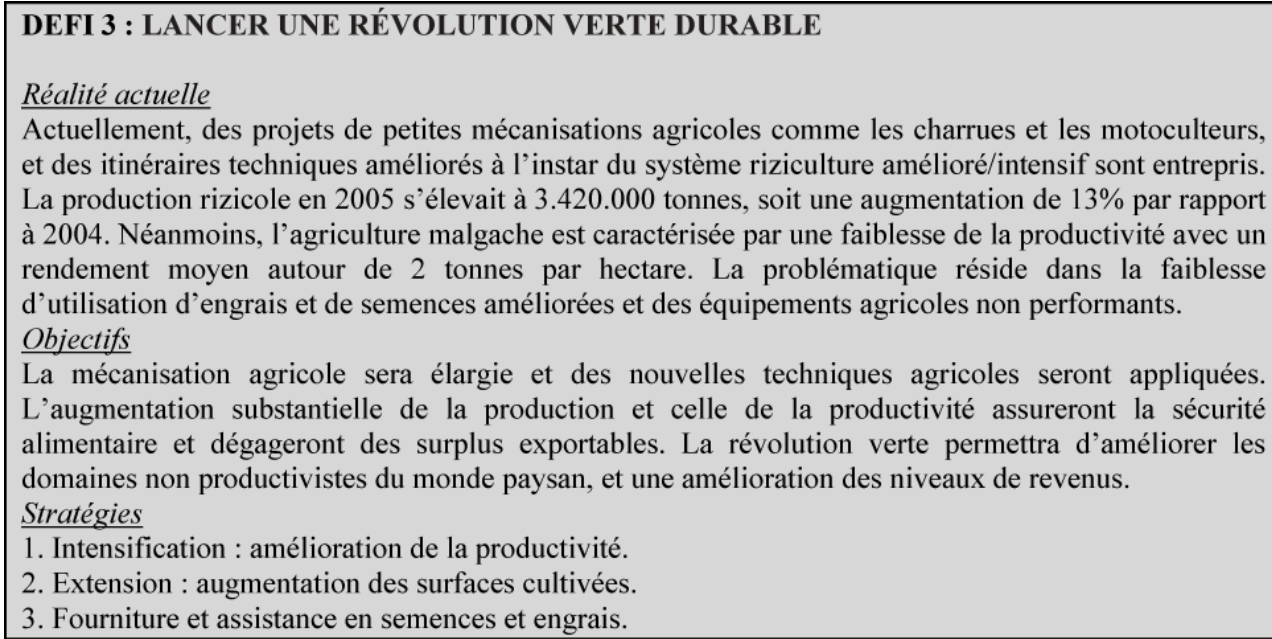

Source : extraits du MAP (www.madagascar.gov.mg/MAP)

Le MAP s'appuie sur une double orientation. D'une part, celle de la vision « Madagascar Naturellement ", document initié au début des années 2000 par le président Ravalomanana pour préciser les priorités de son action ; d'autre part, celle du Millénaire pour le Développement. Il bénéficie du support du Madagascar Challenge Account (MCA), projet signé en 2005 entre Madagascar et les Etats-Unis. Le MCA affiche clairement sa vision du développement, par le passage d'une économie de subsistance à une économie de marché, ce qu'exprime de manière significative l'intitulé du dernier plan quinquennal pour le développement rural (Gouvernement de Madagascar, 2004). Avec la modernisation agricole et l'amélioration de la productivité des exploitations, le MAP fait de l'approvisionnement des marchés extérieurs, en particulier ceux du cadre régional du sud-ouest de l'Océan Indien ${ }^{4}$, une stratégie de croissance de la production agricole et agro-industrielle. Des investissements dans l'agriculture " tournée vers le marché " sont ainsi privilégiés, tout comme " l'appui aux filières porteuses " au sein des Pôles Intégrés de Croissance (PIC) ${ }^{5}$, appui qui doit renforcer l'intégration verticale de ces filières.

La conduite d'une telle modernisation combine l'installation d'unités de production dans l'esprit d'entrepreneurs en agri-business, ce qui correspond à un profil extérieur au monde paysan, et une professionnalisation des paysans. Cette dernière se fait dans deux directions. Elle consiste d'abord en une structuration formelle des producteurs ruraux en organisations par lesquelles transitent désormais les financements agricoles. On observe depuis les années 1990 la mise en place de la Direction d'appui à la professionnalisation des producteurs (niveau Direction du Ministère de l'Agriculture), ainsi que des maisons des paysans (MDP), outil professionnel du développement agricole régional et futures chambres d'agriculture. L'action bénéficie de l'appui d'ONG de développement ou de partenaires associatifs extérieurs, comme Agriculteurs Français et Développement International (AFDI) ou Formation pour l'Epanouissement et le Renouveau de la Terre (Fert). Cette professionnalisation recherche aussi la meilleure efficacité de l'activité. Il s'agit d'améliorer les systèmes de cultures, ce qui exige des agriculteurs "professionnels", impliqués à plein temps dans leur métier. Cette orientation, discutée (Radaniela, 1996), va à l'encontre de la pluriactivité qui accompagne de longue date les stratégies paysannes (Gastellu et Marchal, 1997 ; Rakoto 
Ramiarantsoa, 1995), et qui s'avère particulièrement pertinente en temps de crise (Pierre Bernard et al., 2007). Un regard comparatif établit les méfaits de la spécialisation agricole (Rakoto Ramiarantsoa, 2008) et dénonce le frein qu'une telle situation apporte aux dynamismes ruraux.

\section{...versus agriculture familiale?}

29 L'agriculture familiale, "un mode de vie, une culture, une façon de vivre» (Rasolonirina, 2007), rassemble la majorité des producteurs, petits et moyens paysans malgaches. Rakoto Ramiarantsoa (1995b) la décrit comme une agriculture extensive de paysans pluriactifs, confrontés à un milieu climatique incertain, et qui fonde son système de production sur la combinaison entre différents éléments de son milieu.

30 L'agriculture familiale participe pleinement de la vie des territoires. Elle assure l'occupation des terroirs (les campagnes ne se dépeuplent pas ; l'exode rural caractérise surtout les périodes d'aléas climatiques comme lors des sécheresses dans le grand Sud) et entretient une vitalité des relations entre villes et campagnes (Thibaud, 2006). L'agriculture familiale ne centre pas ses logiques sur la recherche d'un optimum de production. Il est significatif que l'augmentation des coûts de production consécutive à la libéralisation des années 1980 ne se soit pas traduite par une recherche de productivité accrue (Raison, 1994) : il y a au contraire eu réactions de repli sur des pratiques éprouvées car, avec leurs moyens limités et sans autre accompagnement, les producteurs ne peuvent adopter des comportements nouveaux et prendre ainsi des risques. Faute de pouvoir penser à optimiser, ces derniers restent confrontés à des problèmes de divers ordres : manque de moyens, de maîtrise du marché, de sécurité de la production, etc. La priorité actuelle accordée à l'intensification, à l'agri-business, à l'agriculture industrielle est bien éloignée des préoccupations des paysans.

31 L'agriculture familiale est ouverte aux innovations. Elle en est même le vecteur, dans la mesure où les nouveautés répondent à des besoins précis et se trouvent à portée de bourse, comme le montrent les initiatives locales endogènes qui peuvent révolutionner toute une agriculture régionale (Levang, 1993).

32 A Madagascar, les intégrations de techniques de riziculture améliorée vont dans le même sens (Pélissier, 1976 ; Blanc-Pamard et Rakoto Ramiarantsoa, 2000). Un projet en cours, le PSA Tafita ${ }^{6}$, souligne la pertinence de programmes de développement qui savent prolonger les dynamismes paysans. Il s'agit d'une action d'appui aux filières riz, manioc et légumes secs, menée dans trois régions : Matsiatra ambony (hautes terres), Menabe (ouest), et Vatovavy Fitovinany (sud-est). Le programme place les représentants des associations paysannes en situation de jouer un «rôle décisionnel efficace dans la gestion d'un fonds régional pour le financement de services aux agriculteurs $»^{7}$. Cette action prenant fin en 2009, il est prévu de la continuer par une nouvelle structure, celle de Conseil Services Agricoles (CSA) comme interface entre les agriculteurs et les organismes vendant des services aux paysans.

La perspective elle-même est cependant significative. En effet, elle révèle une absence des Maisons Des Paysans pourtant mises en place sous l'impulsion de l'Etat pour aboutir à un tel type de prestations. Cette défection participe des interrogations sur les priorités de développement agricole, dont la révolution verte est un des points de cristallisation (encadrés 2 et 3 ). 
REVOLUTION VERTE DURABLE : est-elle au profit des Agricultures Familiales ? Les medias à Madagascar ont fait un grand ramdam sur la mise en œuvre de la " révolution verte durable ». Les anciens outils agricoles vont être substitués par des matériels modernes. L'utilisation des engrais suffisants, les semences améliorées vont booster les techniques culturales traditionnelles peu productives. Une vraie « révolution»!

Pour la concrétiser, l'Etat malgache va collaborer avec les secteurs privés : fournisseurs d'engrais et importateurs de matériels agricoles modernes. Des banques seront installées dans plusieurs districts de Madagascar.

Mais avec la politique de désengagement de l'Etat, les agricultures familiales risquent de perdre leur importance dans ce nouveau système. Il sera encore difficile pour eux d'accéder aux fruits de cette politique. Aussi, il faut avoir la force pour participer à une révolution, mais est-ce qu'elle est du côté de petits producteurs?

Source : Mouvement paysan Bulletin d'information du syndicat des organisations agricoles, $n^{\circ} 4,2^{e}$ trimestre 2007, p. 3

\section{Encadré 3}

\section{«Révolution verte»}

\section{Révolte des paysans à Ambohibary-Sambaina}

(...) Près de cinq mille personnes ont réagi hier d'une manière assez confuse devant la mairie de la commune rurale d'Ambohibary Sambaina dans la région Vakinankaratra. Ce rassemblement menaçant de paysans (...) A l'origine, selon nos sources, fut la réunion publique organisée dans cette localité d'Ambohibary Sambaina (...) Lors de cette réunion publique, les paysans ont appris de la bouche du chef de Région qu' Ambohibary Sambaina a été choisie comme devant faire l'objet de la politique de la «révolution verte» (...) Ils ont également appris que les reconnaissances devaient s'effectuer incessamment et tout de suite après les travaux dans cette perspective de « révolution verte » allaient commencer.

\section{Confusion}

(...) Progressivement, les paysans apprirent que la « révolution verte » dont il s'agit nécessite le remembrement des terres, donc la disparition des «tahalaka» (diguettes) qui délimitent leurs propriétés. De plus « révolution verte » signifie mécanisation de l'agriculture et intensification des méthodes de culture. (...) tout ceci n'a pas été explicité (...) Les paysans se sont souvenus des intentions des autorités exprimées l'année dernière selon laquelle la plaine d'Ambohibary Sambaina pourrait produire facilement des cultures fourragères au profit d'une entreprise d'élevage et de transformation des produits agricoles. Faisant alors l'amalgame entre ces intentions et cette nébuleuse de "révolution verte », (...) Les paysans d' Ambohibary Sambaina qui vivent de la culture de contre saison (carottes, choux fleurs, navets et autres cultures maraîchères) ne voient pas l'intérêt de cette "révolution verte » ni des bienfaits que pourraient leur apporter le remembrement des terres, la mécanisation et la modernisation de leur système de culture $(\ldots)$

Source : Extraits du quotidien Madagascar Tribune, journal malgache tiré à Tananarive (Jeudi 19 juillet 2007, p. 5, signé : Raw)

Les deux encadrés expriment les risques de confrontation entre deux visions de l'agriculture: celle des paysans qui conçoivent leur activité à travers leurs unités d'exploitation dans le cadre d'un aménagement du terroir, et celle de l'Etat qui fonde sa vision d'abord sur les intérêts nationaux. Cette dernière continue une volonté affichée par la Deuxième République, associant le nécessaire développement des années 2000 à la capacité de production et d'exportation sur le plan alimentaire (livre rouge du président Ratsiraka).L'agriculture de la très grande majorité des ruraux, principale composante de la population, est tenue pour responsable de " pratiques d'exploitation destructives" (Plan d'action Madagascar, 2006). La pression démographique accompagne un accroissement important de la population (la population de l'île, 
estimée à 18 millions d'habitants en 2007, a doublé depuis 1980) ; elle figure parmi les facteurs les plus souvent mentionnés de la dégradation des ressources naturelles à travers une disponibilité limitée en terres cultivables (Plan d'action Madagascar, op. cit.). Il faut cependant relativiser ce constat avec la diversité des situations régionales (Razafimanjato et al., 2001). Les hautes terres centrales et le Sud-Est connaissent une forte occupation humaine (densité largement supérieure à $50 \mathrm{hab} / \mathrm{km}^{2}$ pour une moyenne nationale de 30). Ils s'opposent aux zones faiblement peuplées de l'Ouest, du Sud-Ouest et de l'extrême-Sud. Par ailleurs, densité démographique importante ne rime pas toujours avec dégradation des sols, de nombreux travaux l'ont montré (cf. notamment Tieffen et al., 1993 ; Blanc-Pamard et Rakoto Ramiarantsoa, 2006).

Toujours est-il que chaque catégorie d'agriculture trouve son développement dans un cadre de politiques agricoles spécifiques, plus antagoniques que complémentaires. Rabemanambola (2007) montre ainsi les problèmes du maintien de la petite exploitation paysanne d'élevage à moyen terme, alors qu'actuellement il y a une "prédominance de la livraison par les agriculteurs modestes": les dispositions publiques récentes de la filière laitière excluent en effet peu à peu cette dernière du champ des ressources des paysans.

Le récent retour du thème de l'agriculture au service du développement dans les discours des grands bailleurs de fonds ${ }^{8} e s t$ un fait. Les politiques agricoles retrouvent une légitimité que les Plans d'Ajustement Structurel avaient mise à mal par la libéralisation des marchés. C'est dans ce contexte renouvelé que se situe la nécessité de préciser l'orientation prioritaire de la politique agricole malgache. La situation actuelle pose en effet la question de la viabilité sociale du développement agricole en perspective. On peut la formuler ainsi : un appui à l'intensification agricole, à l'agribusiness dans le cadre d'une économie de marché représente-t-il un choix pertinent pour un pays qui dispose d'une agriculture familiale paysanne majoritaire dont le problème est plus de diversifier les activités et les productions pour répartir les risques, que d'investir dans la recherche de rendements maximaux sur des spéculations limitées?

\section{Conclusion}

37 Les politiques publiques situent clairement le pays dans la voie d'une économie accordant à la libéralisation du marché le rôle prépondérant pour un développement dans le cadre de la mondialisation. Dans le même temps, les injonctions des bailleurs de fonds imposent d'accorder une attention particulière à l'environnement : pour le protéger, le conserver, l'utiliser ; autant de dispositions qui peuvent être réalisées par les acteurs à différents niveaux, suivant leurs objectifs. Le fonctionnement transcalaire du Programme National d'Action Environnemental voit à l'oeuvre ces interférences, aux effets contradictoires : dans un sens comme dans l'autre, environnement et développement ont du mal à cohabiter, alors que le discours du développement durable impose une prise en compte globale. La difficulté est d'autant plus grande que, d'une part, il faut définir les priorités des politiques publiques : la politique agricole veut-elle appuyer l'agriculture familiale, ou développer l'agribusiness ? Faut-il s'adresser à des paysans, ou à des entrepreneurs agricoles ? De même, jusqu'où faut-il que les politiques d'environnement acceptent de transférer les pouvoirs de gestion aux communautés locales riveraines des ressources de la biodiversité ? Peuvent-elles être pleinement 
impliquées dans la dynamique de déforestation évitée (l'Etat malgache s'engage dans cette voie) si elles n'ont pas la propriété des forêts sur lesquelles leur attention permettra d'engranger des crédits Carbone?

D'autre part, ces évolutions se situent dans un contexte qui, progressivement, est passé d'un champ politique et culturel francophone \{les premiers travaux d'inventaire botanique, géologique, pédologique, agronomique (Sourdat, 1996) sont le fait de scientifiques français\} à un champ anglophone \{création de la représentation WWF en 1990, intégration de la Communauté de développement de l'Afrique australe en 2005, l'anglais comme troisième langue officielle depuis 2007\}: le changement ne peut pas être sans conséquence sur les (re)orientations des politiques.

L'ensemble finalement ne dégage pas une idée claire du projet de société, donc de développement, que l'Etat veut mettre en place. Dans cette situation, la place accordée à l'environnement tire parti d'une conjoncture favorable, liée au paradigme du développement durable. Elle n'en comporte pas moins le risque de se trouver en décalage avec les priorités locales et/ou nationales. Ce ne serait alors qu'une opportunité mal utilisée pour le développement des territoires et des sociétés.

\section{BIBLIOGRAPHIE}

ANDRIAMAHEFAZAFY F., MÉRAL, Ph, 2003, Actions des bailleurs de fonds et mise en œuvre des politiques environnementales dans les pays en développement, colloque international « la mondialisation contre le développement ? ", université Versailles-Saint Quentin, 19 p.

AUBERTIN C., 2005, Représenter la nature ? ONG et biodiversité, IRD, Paris, 210 p.

BLANC-PAMARD C., RAKOTO RAMIARANTSOA H., ANDRIANTSEHENO D., 2005, Foncier et territoires entre pouvoirs locaux et politiques publiques environnementales. Pratiques, acteurs, enjeux (corridor betsileo, Madagascar), Cnrs-Ird-Université de Poitiers, $162 \mathrm{p}$.

BLANC-PAMARD C., RAKOTO RAMIARANTSOA H., Madagascar : les enjeux environnementaux In M. Lesourd (coord.), L'Afrique. Vulnérabilités et défis, Nantes, Editions du Temps, p. 354-376.

BLANC-PAMARD C., BOUTRAIS J., 2002, Les temps de l'environnement. D'un sauvetage technique à une gestion locale en Afrique et à Madagascar, Regards sur l'Afrique, Historiens et Géographes $n^{\circ} 379$, congrès UGI de Durban.

DUMOULIN D., RODARY E., 2005, Les ONG, au centre du secteur mondial de la conservation de la biodiversité,in C Aubertin, Représenter la nature ? ONG et biodiversité, IRD, Paris, p. 58-98.

DUPRE G., 1996. Y a-t-il des ressources naturelles ? Cahier des sciences humaines, vol. 32, $\mathrm{n}^{\circ} 1$, p. $17-27$

GASTELLU J.M., MARCHAL J.Y. (éd.), 1997, La ruralité dans les pays du Sud à la fin du XXe siècle, Paris, Orstom, $768 \mathrm{p}$.

GOEDEFROIT S., REVERET J-P (textes réunis et présentés par), 2007, Etudes Rurales n 178 , Quel développement à Madagascar? Paris, EHESS, $284 \mathrm{p}$. 
GOODMAN S. M., BENSTEAD, J. P. (sous la direction de), 2004, The Natural History of Madagascar. University of Chicago Press, 1728 p.

GOUROU P., 1982, Terres de bonne espérance : le monde tropical. Paris, Plon, 456 p.

GOUVERNEMENT DE MADAGASCAR, Ministère de l'Agriculture, de l'Élevage et de la Pêche, 2004, Plan Directeur quinquennal pour le développement rural : une approche de marché pour la lutte contre la pauvreté en milieu rural à Madagascar, avril 2004 à mars 2009. Antananarivo, Madagascar, $68 \mathrm{p}$.

GUILLAUMET J-L., 1984) The vegetation : an extraordinary diversity in Jolly, A. Oberlé P., et Albignac E.R. (eds), Key environments .Madagascar. Pergamon press oxford p. 27-54.

HUFTY M., 2001, La gouvernance internationale de la biodiversité, Etudes internationales, vol. 32, $\mathrm{n}^{\circ} 1$, mars, p. 5-29.

KARSENTY A., MARIE J., 1998, Les tentatives de mise en ordre de l'espace forestier en Afrique centrale in ROSSI G., LAVIGNE DELVILLE P., NARBEBURU D. (dir) Sociétés rurales et environnement. Gestion des ressources et dynamiques locales au Sud. Paris : Karthala/Regards/GRET, p. 153-175.

KULL C. A., 1996 : The evolution of conservation efforts in Madagascar. International Environmental Affairs, 8 (1) ; p. 50-86.

LEVANG P., 1993, Savah tada hujan, ou comment étendre l'effet bas-fond à la riziculture pluviale. Bas-Fonds et riziculture. Actes du séminaire d'Antananarivo, 9-14 décembre 1991 (éd. sc. M. Raunet), Montpellier, p. 83-90

MICHON G., 2002. Du discours global aux pratiques locales, ou comment les conventions sur l'environnement affectent la gestion de la forêt tropicale ? in Martin J.Y. (éd.), Développement durable? Doctrine, pratiques, évaluations, IRD éditions, Paris, 183-203.

PELISSIER P., 1976, Les riziculteurs des hautes terres malgaches et l'innovation technique. Cahiers ORSTOM, série Sciences Humaines, vol. 13, n 1, p. 41-56

PELISSIER P., 1995, Campagnes africaines en devenir, Ed. Arguments, Paris, 318 p.

PIERRE BERNARD A., RAMBOARISON R., RANDRIANARISON L., RONDRO-HARISOA L., 2007, Les implications structurelles de la libéralisation sur l'agriculture et le développement rural. Première phase : Synthèse Nationale Madagascar, Programme RuralStruc, EPP-PNDR, Banque Mondiale, Coopération Française, APB Consulting, 190 p.

PLAN d'ACTION MADAGASCAR 2007-2012, 2006, Un plan audacieux pour le Développement Rapide, 112 p. (www.madagascar.gov.mg/MAP)

RABEMANAMBOLA M.F., 2007, LE TRIANGLE LAITIER MALGACHE. Contribution à l'étude d'une filière alimentaire et de son inscription spatiale dans un pays en voie de développement. Université de Clermont-Ferrand II, thèse DNR, 375 p.

RADANIELA H. N. A., 1996, La professionnalisation des paysans est-elle une réponse aux problèmes de la paysannerie à Madagascar? Le cas de l'association paysanne Fifata à Ampamelomana dans le Vakinankaratra, mémoire Capen, Ecole Normale Supérieure, Antananarivo, 83 p.

RAISON J-P. (coord.)., 1994, Paysanneries malgaches dans la crise. Paris, Karthala, 376 p.

RAISON J-P., 1995, l'Afrique du Sud et l'Océan Indien : une approche géopolitique, Communication au colloque France-Afrique du Sud-Océan Indien, 14 p. multigr. 
RAKOTO RAMIARANTSOA H, 1995a, Chair de la terre, oeil de l'eau... : paysanneries et recompositions de campagnes en Imerina (Madagascar). Paris : Orstom, coll. A travers champs, $370 \mathrm{p}$.

RAKOTO RAMIARANTSOA H, 1995b, Regards, ou "c'est en écartant les feuilles que l'on trouve les racines", mémoire HDR, Université de Paris X, 161 p.

RAKOTO RAMIARANTSOA H, 1997a, Paysanneries mérina des hautes terres centrales malgaches : une ethno-agronomie déphasée ? Cahiers d'Outre-mer, Bordeaux, n 197, p.43-56.

RAKOTO RAMIARANTSOA H, 1997b, Agir sans comprendre les pratiques paysannes, ou un aspect du problème des projets de conservation du milieu sur les hautes terres tropicales. Montagnes et hautes terres africaines face aux changements économiques dans le monde. Quatrième colloque international de l'Association des Montagnes Africaines - African Highlands Initiatives.Tananarive, 26 mai- ${ }^{\text {er }}$ juin. 7 p.

RAKOTO RAMIARANTSOA, H, SAMYN, J-M., 2004, Arrimer le global au local ou le développement durable pour qui ? L'exemple de la gestion contractualisée de la forêt de Merikanjaka. Mondes en développement, Paris, vol. 32, $\mathrm{n}^{\circ}$ 127. p. 89-97

RAKOTO RAMIARANTSOA H, 2008, De nouvelles questions, vers un partenariat élargi. Les nouvelles mutations du monde rural : question foncière, mobilité des hommes, circulation des produits, rapport scientifique final, Action Incitative de l'université de Poitiers, Université de Poitiers, p. $79-84$

RAKOTO RAMIARANTSOA H, 2008 (à paraître), Développer le territoire, en signant pour la forêt : la Gelose " activée » dans la commune rurale de Merikanjaka (district de Manjakandriana, bordure orientale de l'Imerina), In Actes du programme de recherche "Is Community-Based Management of Forest Resources the Management System of the Last Resort in Developing Countries ? A Case Study in Madagascar", Université Roskilde, Danemark, 17 p. + annexes.

RAMAMONJISOA B., 2004, Origines et impacts des politiques de gestion des ressources naturelles à Madagascar, Schweiz. Z. Forstwes, 155, $10 \mathrm{p}$.

RANDRIANASOLO E., RASOLO K., RAKOTO RAMIARANTSOA H., 1997,Bilan - évaluation du projet de conservation des sols du bassin versant de Mahakamo (Anjozorobe), in Bilan et évaluation des travaux et réalisations en matière de conservation des sols à Madagascar. Projet Conservation des Sols, République de Madagascar, ONE-FOFIFA-ANAE-CIRAD, $60 \mathrm{p}$.

RASOLONIRINA M, 2007, Soa s'organise pour défendre les intérêts de l'agriculture familiale, Mouvement Paysan, bulletin d'information du syndicat des organisations agricoles, $n^{\circ} 4$, p. 1

RAZAFINJATO O, 1996, Déforestation et humanisation : paysans et paysages du Nord-Est de l'Imerina, ENS, Université d'Antananarivo, mémoire de Capen, $100 \mathrm{p}$.

ROSSI G., LAVIGNE DELVILLE P., NARBEBURU D. (dir) , 1998, Sociétés rurales et environnement. Gestion des ressources et dynamiques locales au Sud. Paris : Karthala/Regards/GRET, $402 \mathrm{p}$.

SAHLER K. 2005, Approche contractuelle de la gestion des ressources naturelles à Madagascar. Politiques environnementales et recompositions rurales. Mémoire DEA, Université Toulouse Le Mirail, École Nationale Supérieure Agronomique de Toulouse, École Nationale de Formation Agronomique, 132 p.

SOURDAT, M., 1996, Une évaluation agronomique des terres de Madagascar - 1897-1900. L'étude d'A. Müntz et sa correspondance avec A. Grandidier, in Etude et gestion des sols, 3.3, Orstom, p. $207-214$ 
THIBAUD B., 2006, « Ouverture des terroirs et stratégies paysannes renouvelées dans les campagnes du sud-ouest malgache ». Revue internationale des Sciences Humaines et Sociales, Yaoundé, Cameroun, vol. 1, nº 1, p. 391- 409.

UNIVERSITÉ D'ANTANANARIVO, Fofifa, Inra, Cirad, 2007, Agriculture intra et périurbaine dans

l'agglomération d'Antananarivo, CD, projet multidisciplinaire ADURAA.

\section{NOTES}

1. Citons comme autre exemple l'appui accordé à la Commune Urbaine d'Antananarivo par le programme des Nations Unies pour les établissements humains (Onu-habitat) et par la Banque Mondiale, afin d'élaborer une «stratégie de développement de l'agglomération d'Antananarivo Développement des infrastructures, amélioration des services urbains et réduction de la pauvreté urbaine ».

2. Dans cette perspective, le Président de la République, à son retour de la conférence internationale de Durban, a annoncé un triplement de la surface des Aires Protégées malgaches)

3. Le PNAE débute officiellement en 1990 et prévoit une déclinaison en trois phases:

Programme Environnemental 1 (prévu de 1991 à fin 1996) : mise en place des fondations institutionnelles, et actions de conservation les plus urgentes

Programme Environnemental 2 (prévu de 1997 à 2002) : intensifier les actions du PE 1 et mettre en oeuvre la gestion décentralisée des ressources naturelles

Programme Environnemental 3 (prévu de 2003 à 2008) : « développer le réflexe environnemental chez tous les acteurs »

4. Par exemple, Madagascar intègre le Southern Africa Development Community en 2005 ; l'ensemble représente un marché de plus de 200 millions de consommateurs.

5. Ces zones présentent des caractéristiques telles qu'une densité démographique "élevée », la présence d'infrastructures routières permettant l'évacuation des produits et l'approvisionnement en intrants,le dynamisme d'organismes de développement (ONG, projet...) ; l'axe Antananarivo-Antsirabe est un exemple de ferment de tels pôles.

6. Projet Sécurité Alimentaire, avec soutien financier de l'Union Européenne

7. prospectus de présentation des équipes du Psa-Tafita Menabe, Matsiatra ambony et Vatovavy Fitovinany (Projet Sécurité Alimentaire, Fianarantsoa, Haute Matsiatra)

8. Citons le rapport 2007 du Comité d'Aide au Développement de l'OCDE; le rapport 2008 de la Banque Mondiale sur le développement dans le monde ; la déclaration (juin 2008) du Secrétaire Général des Nations Unies sur la nécessité d'accroitre la production alimentaire de $50 \%$ pour faire face aux besoins d'ici 2030.

\section{RÉSUMÉS}

De la République humaniste et écologique souhaitée par le président Ratsiraka au développement rapide et durable prôné par Marc Ravalomanana, Madagascar voit la question de son développement, centrale pour une nation classée dans le groupe des Pays Pauvres et Très Endettés, désormais liée à des préoccupations environnementales. Une telle orientation accompagne le choix malgache de s'engager, à partir des années 1980, dans les perspectives 
proposées par la Banque Mondiale. Les thèmes prioritaires de développement durable, de bonne gouvernance, de lutte contre la pauvreté..., le soulignent. En même temps, le pays entend mener la politique de sa géographie dans cet engagement. Y trouve-t-il son compte? A travers une réflexion sur les politiques mises en œuvre au plan de l'agriculture et au plan de l'environnement, deux sujets au cœur des problématiques de développement d'un pays très rural, l'article analyse les tensions générées par les interférences des différents niveaux (réalités locales, décisions nationales, injonctions supranationales) auxquels se rattachent les notions de développement et d'environnement. Il relève l'absence d'un projet de société qui articule clairement ces différentes échelles afin que, au-delà du paradigme porteur de développement durable, l'environnement soit au service du territoire et de la société malgaches.

From the humanist and ecological Republic wished for by President Ratsiraka to the rapid and sustainable development advocated by Marc Ravalomanana, the question of development, major for Madagascar, classified among the Highly Indebted Poor Countries, is henceforth linked to environmental concerns. This orientation has accompanied the Madagascan choice to embark on the proposals of the World Bank, since the 1980s, and is outlined by priority issues such as good governance, sustainable development, and struggle against poverty. In the same time, Madagascar intends to carry on the policy of its geography to achieve this commitment. Does the country do well out of it? Through a reflection on the different policies set up for agriculture and environment, two central issues in the development of a rural country, the paper analyses the tensions resulting from interferences at different levels (local realities, national decisions, supra national injunctions) with which the notions of development and environment are connected. It brings out the lack of a society plan which clearly links up these different scales so that, beyond the booster paradigm of sustainable development, the environment be at the service of the Madagascan territory and society.

\section{INDEX}

Mots-clés : développement rural, environnement, politique publique, territoire

\section{AUTEUR}

\section{HERVÉ RAKOTO RAMIARANTSOA}

Hervé Rakoto Ramiarantsoa (herve.rakoto-ramiarantsoa@mshs.univ-poitiers.fr) géographe, professeur à l'université de Poitiers. Il est actuellement en accueil à l'IRD, UR 199 (Dynamiques socio-environnementales et gouvernance des ressources), au sein de l'équipe « Politiques de l'environnement » du site d'Orléans. Ses recherches s'orientent dans trois directions : la gestion des ressources, les recompositions sociales et territoriales liées aux politiques environnementales, les ruralités. Son livre « Chair de la terre, œil de l'eau... : paysanneries et recompositions de campagnes en Imerina » (Ird, collection A travers Champs, 1995) a été primé du prix Tropiques. Il est par ailleurs le coordinateur principal de l'ouvrage « Ruralités Nords-Suds : inégalités, conflits, innovations » (452p. ; à paraître en novembre 2008 chez L'Harmattan, collection Itinéraires géographiques). Ses trois dernières publication sont :

- La gestion contractualisée des forêts en pays betsileo et tanala (Madagascar). Les effets biaisés d'un modèle universel, in Cybergeo, Environnement, Nature, Paysage, (mis en ligne le 04 juillet 2008); en collaboration avec Chantal Blanc-Pamard

- Normes environnementales, transferts de gestion et recompositions territoriales en pays 
betsileo (Madagascar). La gestion contractualisée des forêts, in Natures Sciences Sociétés, 2007,15, p. 253-268 ; en collaboration avec Chantal Blanc-Pamard

- Nuages, vents et pluies : scruter le « visage du firmament » en Imerina, 2007, In Les Cahiers d'Outre-Mer, Bordeaux, n²40, p. 319-340 ;en collaboration avec Daniel Peyrusaubes 